\title{
Skin detection based on thresholding in RGB and hue component
}

\begin{abstract}
This paper presents a skin detection of color image using thresholding in hue component of HSV (Hue, Saturation, and Value) and RGB (Red, Green and Blue) color spaces. The reason for choosing hue component for thresholding compared to two other components is hue gives the best result for skin detection. The process of thresholding in hue component is done in order to get candidate pixels of skin regions. Then, the rectangular box of these blob regions are estimated and used to do thresholding in RGB color space where the mean and standard of each rectangular box is calculated. This method, is similar to adaptive thresholding technique in grayscale image processing, is able to detect the skin region of the test images accurately and with an acceptable robustness.
\end{abstract}

Keyword: Color; Skin; Color space; Thresholding 